\title{
Social media and healthcare quality improvement: a nascent field
}

\author{
Megan L Ranney, ${ }^{1}$ Nicholas Genes ${ }^{2,3}$
}

\begin{abstract}
${ }^{1}$ Emergency Digital Health Innovation Program, Department of Emergency Medicine, Rhode Island Hospital/Alpert Medical School, Brown University, Providence, Rhode Island, USA ${ }^{2}$ Department of Emergency Medicine, Icahn School of Medicine at Mount Sinai, New York, New York, USA ${ }^{3}$ Department of Genetics \& Genomic Science, Icahn School of Medicine at Mount Sinai, New York, New York, USA
\end{abstract}

\section{Correspondence to}

Dr Megan L Ranney, Emergency Digital Health Innovation Program, Department of Emergency Medicine, Rhode Island Hospital/Alpert Medical School, Brown University, 593 Eddy St, Claverick 2, Providence, RI 02903, USA; mranney@ lifespan.org

Accepted 17 November 2015 Published Online First

11 December 2015

\section{SLinked}

- http://dx.doi.org/10.1136/ bmjqs-2015-004489 - http://dx.doi.org/10.1136/ bmjqs-2015-004309

\section{CrossMark}

$$
\begin{aligned}
& \hline \text { To cite: Ranney ML, } \\
& \text { Genes N. BMJ Qual Saf } \\
& \text { 2016;25:389-391. }
\end{aligned}
$$

Two articles ${ }^{12}$ describe the use of social media to describe or potentiate healthcare quality improvement. Taken together, these articles point to an exciting-but still nascent-trend. We hope that these two pilot studies will be taken as a call to future research rather than as definitive reports.

The first article ${ }^{1}$ describes the proportion of emergency department (ED) patients who consent for researchers to download their Facebook and Twitter data. The novelty of this study lies in its underlying premise: accessing patients' social media data could, in theory, permit better awareness of patients' health status and risks, and thereby permit real-time interventions and improved patient engagement. To our knowledge, this study is the first to show the acceptability, feasibility and limitations of accessing patients' social media data. It thereby gives hope that incorporating social feeds into healthcare may be possible.

This study had three important, and surprising, findings. First, the authors report that $52 \%$ of ED patients reported using Twitter or Facebook. This fraction is much lower than found in other urban EDs (eg, 67\% of ED patients in a 2012 manuscript $)^{3}$ or in national surveys $(62 \%$ of all US adults, according to recent Pew $\left.\mathrm{data}^{4}\right)$. Second, of those patients using social media, 53\% agreed to participate in the study, and approximately two-thirds of those agreed to have data downloaded. These statistics could be interpreted as encouraging-representing a higher-than-expected proportion of ED patients who are willing to share their social media feeds with researchers. It could also reflect a limitation to the applicability of this method for healthcare in general: it would be difficult to argue for implementation on a large scale, if only $37 \%$ of those with social media feeds agree to sharing their data. Finally, those participating in the study were less likely to be injured or have psychiatric complaints compared with the general ED population; these categories of patients seem most likely to benefit from insights gleaned by integrating social feeds into health records. These results, in toto, lead us to wonder: why did $50 \%$ of social media users refuse to even participate, and why was there differential participation according to chief complaint? What could be done to increase participation in the future? To what degree would these findings translate to other EDs, and to non-research applications? Future work should better characterise the refusers, and should be sure to capture the full range of online social networking platforms, to minimise the inherent bias in this strategy.

The second article, 'Measuring patientperceived quality of care in U.S. hospitals using Twitter'2 extends others' work using social media to measure or evaluate healthcare. Twitter has previously been shown to provide real-time monitoring of conditions such as viral and foodborne illness, ${ }^{5}$ mental illness ${ }^{6}$ and substance use. $^{7} 8$ The study by Hawkins et $a l,{ }^{2}$ showing a correlation between patient sentiment and readmission rates, has findings that are similar to Kilaru et al's ${ }^{9}$ recent article in this journal that analysed Yelp reviews. While these methods provide insight into a variety of patient characteristics and behaviours, they are nonetheless limited and subject to misinterpretation. Analysing Twitter and other forms of social media for real-time feedback on care may be faster and cheaper than traditional surveys or US Hospital Consumer Assessment of Healthcare Providers and Systems scores, allowing administrators and researchers to quickly gauge the impact of new quality improvement initiatives. However, Twitter is but one data stream, and is currently the least used form of social media (behind Facebook, LinkedIn, Pinterest and 
Instagram). ${ }^{10}$ As we have previously cautioned, any conclusions drawn from a sole social media source are subject to immense selection bias. ${ }^{11}$

We also caution that the relationship between correlation and causation is particularly thorny with social media. We recall the conclusion by Eichstaedt, Merchant et al: ${ }^{12}$ while tweets reflecting anger, negativity and negative social relationships correlate with cardiovascular deaths, the people tweeting are very unlikely to be the people dying. In the study by Hawkins et $a l,{ }^{2}$ positive sentiment is correlated with decreased readmission, even though it's very unlikely that the tweeters are the same group as the readmitted. While it's tempting to conclude that positive tweets are a reflection of superior hospital care and thus decreased readmissions, other possibilities merit investigation (positive tweets may reflect increased medication adherence, or a tendency to avoid complaints that would prompt readmission). Relatedly, the finding that overall sentiment does not correlate with the number of hospital-initiated tweets was notable; hospitals, like other institutions, should realise that a public relations campaign can't drown out an individual's complaints.

Another major limitation for both of these articles is that we still have problems accurately interpreting the meaning of social media. Machine learning and natural language processing are bound to miscategorise such small snippets of text with varying frequency. By comparison, hand-coding (as in Padrez $e t a l^{1}$ ) is time-intensive and subject to bias from extrapolation from a small number of coded posts. Analyses such as these should therefore prompt further inquiry to tease out a real relationship, rather than being taken as the last word on the subject.

Finally, both articles touch on-but do not provide answers to-the spectre of privacy. In the first paper, among the 50\% willing to engage in discussion, privacy was the primary reason for people refusing to share their data. The authors note that in general, public supports purpose of using social media data for health research, accepting it without consent (compared with their lack of support for marketing research, done with their consent)-but this paper suggests attitudes change when it's an individual's data that's being requested, during that individual's emergency. These findings are similar to those of others' findings regarding patients' privacy preferences for electronic health information. ${ }^{13}$ Although many posts (particularly on Twitter) are already publicly accessible, these posts are also often identifiable; tying their online persona to a medical encounter is perhaps a bridge patients will be unwilling to cross. Further work-particularly qualitative inquiry-on this subject needs to be conducted.

All of these limitations aside, we are enthused by these papers. Together, they help lay the foundation for further efforts integrating social media into electronic health record (EHR) and healthcare. We can imagine a future state where hospital administrators review patient comments about their care on social platforms in real time, just as marketers monitor brand mentions today. We can also imagine a reality in which primary care doctors and insurers routinely scrutinise patients' social media writings, looking for keywords, descriptions of behaviours, or friend lists that suggest declining health status or increased health risks. Stakeholders may be able to use social media alongside history and physical exam to raise or lower the thresholds for testing, or interventions. Even more intriguingly, interventions could be initiated over social media, such as automated (but personalised) reminders or warnings, when a patient's social activity seems to veer into riskier territory. ${ }^{14}$ These possibilities are exciting and ethically fraught.

While these questions and scenarios become increasingly speculative, they are all possible-if it turns out that collecting this social network data yields new, real, actionable insights on patient risks. Thus far, however, that's still a big 'if'-though these authors are taking that first step in collecting and analysing this new source of data.

Twitter Follow Megan Ranney at @meganranney and Nicholas Genes at@nickgene

Competing interests None declared.

Provenance and peer review Commissioned; internally peer reviewed.

\section{REFERENCES}

1 Padrez KA, Ungar L, Schwartz HA, et al. Linking social media and medical record data: a study of adults presenting to an academic, urban emergency department. BMJ Qual Saf 2016;25:414-23.

2 Hawkins JB, Brownstein JS, Tuli G, et al. Measuring patient-perceived quality of care in U.S. hospitals using Twitter. BMJ Qual Saf 2016;25:404-13.

3 Ranney ML, Choo EK, Wang Y, et al. Emergency department patients' preferences for technology-based behavioral interventions. Ann Emerg Med 2012;60:218-27.e48.

4 Perrin A. Social Media Usage: 2005-2015. October 2015. http://www.pewinternet.org/2015/10/08/ social-networking-usage-2005-2015/ (accessed 13 Nov 2015).

5 Signorini A, Segre AM, Polgreen PM. The use of Twitter to track levels of disease activity and public concern in the U.S. during the influenza A H1N1 pandemic. PLoS One 2011;6: e19467.

6 Jashinsky J, Burton SH, Hanson CL, et al. Tracking suicide risk factors through Twitter in the US. Crisis 2014;35:51-9.

7 Hanson CL, Burton SH, Giraud-Carrier C, et al. Tweaking and tweeting: exploring Twitter for nonmedical use of a psychostimulant drug (Adderall) among college students. J Med Internet Res 2013;15:e62.

8 Chary M, Genes N, McKenzie A, et al. Leveraging social networks for toxicovigilance. J Med Toxicol 2013;9:184-91. 
9 Kilaru AS, Meisel ZF, Paciotti B, et al. What do patients say about emergency departments in online reviews? A qualitative study. BMJ Qual Saf 2016;25:14-24.

10 Duggan M, Ellison NB, Lampe C, et al. "Social Media Update 2014”, Pew Research Center. January 2015. http://www.pewinternet.org/2015/01/09/social-mediaupdate-2014/

11 Ranney ML, Peimer CA. Online emergency department ratings, patient satisfaction and the age-old issue of communication. BMJ Qual Saf 2016;25:1-2.
12 Eichstaedt JC, Schwartz HA, Kern ML, et al. Psychological language on Twitter predicts county-level heart disease mortality. Psychol Sci 2015;26:159-69.

13 Grande D, Mitra N, Shah A, et al. Public preferences about secondary uses of electronic health information. JAMA Int Med 2013;173:1798-806.

14 Pechmann C, Pan L, Delucchi K, et al. Development of a Twitter-based intervention for smoking cessation that encourages high-quality social media interactions via automessages. J Med Internet Res 2015;17:e50. 